\title{
ABOMASAL DILATATION AND EMPTYING DEFECT IN A SUFFOLK EWE
}

\author{
SILVA, M.A. ${ }^{1}$; DECONTO, I. ${ }^{2}$; FERRARI, M.V. ${ }^{2}$; VAN DER VINNE, R. $^{3}$ \\ ${ }^{1}$ Fundação Faculdades "Luiz Meneghel"; \\ ${ }^{2}$ Universidade Federal do Paraná; \\ ${ }^{3}$ Curso de Pós-Graduação em Ciências Veterinárias, Universidade Federal do Paraná.
}

In June of 2000, at the Large Animals Clinics of the UFPR Veterinary Hospital care was taken of a Suffolk PO sheep of about $40 \mathrm{~kg}$ weight showing wool fall, progressive weight loss, anorexia and kyphosis when standing up. No observed significant alterations in the breathing frequency (26 breaths/minute) and heart frequency ( 80 beats/minute)were observed at the physical examination. However hypermotility of the rumen (4 ruminations/minute) besides a light hypothermia $\left(38.5^{\circ}\right.$ C) were observed. The ocular mucous membranes were pale. The animal expelled a small daily volume of liquid fecal material in which no presence of fibers were observed by microscopic analysis. Through abdominal palpation it was noticed, in the inferior right portion of the abdomen, a hard mass that when touched the animal reacted demonstrating painful sensibility. The clinical pathology revealed anemia (PCV 23\% and total count of erythrocytes 3,9 million $/ \mu \mathrm{L}$ ); leukopenia (5300 leukocytes/ $\mu \mathrm{L}$ ) and lymphopenia (1370 lymphocytes/ $\mu \mathrm{L}) ;$ increase in the serum levels of total bilirubin $(0,99 \mathrm{mg} / \mathrm{dL})$ and indirect bilirubin $(0,74 \mathrm{mg} / \mathrm{dL})$. The fecal examination revealed presence of estrongilíd eggs $(400 \mathrm{e} / \mathrm{g})$ and oocysts of protozoa, but negative for the presence of Fasciola hepatica eggs. The sheep stayed four days at the Hospital in observation receiving support therapy with vitamins $B, A, D$ and $E$, and antibiotic therapy (penicillin $25000 \mathrm{lU} . \mathrm{kg}-1$ ). At the fifth day of hospitalization, exploratory laparotomy was proceeded, abomasal dilation being confirmed. Soon after, took place the abomasotomy for the removal of the compacted content. After the surgery, the animal received penicillin (25000 Ul.kg-1 every 12 hours) and enrofloxacin (7,5 mg.kg-1 every 24 hours) for five days. Besides the antibiotic therapy, the animal received for four days: metoclopramide $(0,75$ mg.kg-1 every 12 hours), neostigmine (0,025 mg.kg-1 every 24 hours), cimetidine (2,5 mg.kg-1 every 12 hours) and flunixin meglumine (1,25 mg.kg-1 every 24 hours). The patient showed an improved clinical evolution being dismissed from the Hospital 10 days after the accomplishment of the surgery. Due to its clinical implications, abomasal dilation and the consequent emptying defect is an illness of high letality, due to the evolution of the disease and also for the lack of correct diagnosis. The abomasotomy in skilled time and the correct nursing in the postoperative, are indispensable factors for the clinical success in this illness.

Key Words: Abomasal dilatation, abomasotomy, Suffolk, ewe. 